KANSAS JOURNAL of MEDICINE

\section{An Overlooked Cause of Rifampin Resistance}

Yasmine Hussein Agha, M.D. ${ }^{1}$, John Millard, Pharm.D. ${ }^{2}$, Maha Assi, M.D., MPH ${ }^{1,3}$

${ }^{1}$ University of Kansas School of Medicine-Wichita, Wichita,

KS, Department of Internal Medicine

Robert J. Dole VA Medical Center, Wichita, KS

${ }^{2}$ Department of Pharmacy

${ }^{3}$ Department of Infectious Disease

Received April 27, 2020; Accepted for publication. June 8, 2020; Published online August 17, 2020

\section{INTRODUCTION}

Staphylococcus and Streptococcus sp. are the most common pathogens causing a wide range of complications following joint replacement procedures. ${ }^{1}$ These microorganisms grow in biofilms attached to the prosthetic implants, which allows them to evade the host's immune response and resist antibiotics. ${ }^{2}$ As a result, the rate of joint failure has been increasing steadily, which prompted research into the most optimal approach for treating prosthetic joint infections. Treatment usually consists of surgery and antibiotics capable of penetrating bone and biofilm producing pathogens. In this report, we focus on antibiotic therapy in prosthetic joint infections secondary to methicillin-resistant Staphylococcus aureus (MRSA). We present a case of relapsing MRSA bacteremia associated with development of rifampin resistance in a patient with L3-L4 discitis, ischial and lumbar osteomyelitis, and psoas abscess.

\section{CASE REPORT}

A male in his late 80 's presented with sepsis. He was febrile, tachycardic, and lethargic. He had a history of left total knee and hip replacement five years prior. He also had left femur fractures a year prior to presentation and underwent fixation with intramedullary rods in the femoral neck and shaft. Physical examination was remarkable for a deep lumbosacral ulcer. The left knee and hip did not show any signs of infection or joint effusion.

Further work-up revealed that the white blood cell count was 18,000 cells $/ \mathrm{mm}^{3}$ and the erythrocyte sedimentation rate was 120 $\mathrm{mm} / \mathrm{hr}$. His urinalysis was positive for nitrites, leucocyte esterase, bacteria, and white blood cells. Laboratory testing was otherwise unremarkable. His chest x-ray did not reveal any source of infection. Magnetic resonance imaging was consistent with right ischial and lumbar spine osteomyelitis, L3-L4 discitis, and psoas muscle infection. The patient was started on vancomycin and cefepime on the first day of admission.

Initial blood cultures were positive for MRSA, with persistent growth on two repeat blood cultures drawn 48 hours apart on day three and day five of admission. The urine culture did not grow any organism. Transesophageal echocardiogram (TEE) was negative for vegetations. Cefepime was discontinued. Despite treatment with broad spectrum antibiotics, the patient was persistently febrile.
Rifampin was started on day five owing to concerns for hardware infection. All culture susceptibilities showed sensitivity to rifampin and vancomycin with mean inhibitory concentration (MIC) less than $0.5 \mu \mathrm{g} / \mathrm{ml}$ and $1 \mu \mathrm{g} / \mathrm{ml}$, respectively. The last blood culture set drawn on day seven had no growth. Of importance, vancomycin trough drawn on day five was $9.1 \mathrm{mcg} / \mathrm{mL}$, below therapeutic range. Dose was adjusted and trough was adequate on day seven of admission $(21.4 \mathrm{mcg} / \mathrm{ml})$. A bone scan did not show any uptake in the joints or hardware. Since prosthetic joint infection was ruled out, rifampin was discontinued. A bone biopsy was not done due to the lack of resources at the hospital; therapy was based on blood cultures.

Upon resolution of symptoms and stability, the patient was discharged to a transitional living center to complete six weeks of intravenous vancomycin. The patient presented again two days following his discharge with sepsis, despite compliance with intravenous antibiotic therapy. Blood cultures grew MRSA and susceptibilities showed resistance to rifampin with mean inhibitory concentration greater than $32 \mu \mathrm{g} / \mathrm{ml}$. The patient did not want to continue intravenous antibiotic therapy. He elected to go home with comfort care.

\section{DISCUSSION}

Treatment of prosthetic joint infections secondary to MRSA usually begins with parenteral vancomycin, a broad-spectrum antibiotic that inhibits cell wall formation and oral rifampin that inhibits bacterial RNA-polymerase. ${ }^{2-4}$ No specific guidance exists as to when rifampin should be initiated in patients with prosthetic related infections. Improved outcomes were seen in animals when comparing vancomycin vs. vancomycin/rifampin combination; however, the investigators did not address when rifampin was started. ${ }^{5}$ Rifampin monotherapy is not recommended due to rapid development of resistant strains following mutations in the rpoB gene. ${ }^{6}$ Despite dual antibiotics therapy, treatment failure occurs, causing significant morbidity to the patients and frequent hospital readmissions.

MRSA as a pathogen is a major risk factor predicting poor outcome after treatment. ${ }^{7}$ Other causes included failure to identify a second pathogen, the choice of surgery (debridement antibiotics and implant retention, one step or two step exchange), patient dependent risk factors such as higher body mass index, cardiac insufficiency, and purulence at the site of infection or inability to use vancomycin due to chronic kidney disease, and increased risk for nephrotoxicity. ${ }^{8,9}$

Important causes of rifampin resistance include monotherapy, combination with oral antibiotics with low bioavailability, or narrow spectrum antibiotics. ${ }^{6}$ In this report, an antibiotic-related factor was highlighted that might have been overlooked and not accounted for in patients with rifampin resistance. In this patient, at the time rifampin was given, vancomycin was not within therapeutic levels. There was a 48-hour window during which the patient was being treated with rifampin only secondary to sub-therapeutic vancomycin levels. This might have contributed to rifampin resistance. Perhaps, rifampin only should be initiated after a therapeutic vancomycin level has been obtained to decrease the risk of resistance. This particular case was unique by providing a serendipitous insight into how quickly rifampin resistance can develop in vivo despite the patient being treated with vancomycin. Initiating vancomycin and rifampin prior to a known 
therapeutic level of vancomycin could be exposing patients to the adverse event profile of rifampin without the therapeutic benefits of this combination secondary to rapid rifampin resistance development.

Furthermore, resistance could develop and remain undetected in cases where initial clearance of bacteremia occurs with vancomycin. Bacterial heterogeneity may exist within a single bacterial population and the patient could have been infected with multiple MRSA strains. The predominant strain may have been sensitive to rifampin and first detected in blood cultures while the other strain differed phenotypically by expressing rifampin resistance. Prior to treatment, the inoculum with the rifampin resistance strain was not the strain detected in cultures. Based on the outcome seen in our patient, treatment during the rifampin-only window could have allowed the resistant strain to become predominant while the sensitive strain was being inhibited or eradicated.

Coiffier et al. ${ }^{3}$ suggested that starting treatment initially with vancomycin only would decrease the bacterial load, which would decrease the risk of resistance to rifampin. Lora-Tamayo et al. ${ }^{10}$ highlighted the higher rate of successful treatment of prosthetic joint infection following biofilm and inoculum removal during debridement. The Infectious Disease Society of America (IDSA) recommends initiating antibiotic therapy in patients with device related infections along with surgical debridement. ${ }^{4}$ However, there was no mention of whether antibiotics should be given before or after debridement. It might be safer to attempt decreasing bacterial load with vancomycin and surgical debridement prior to initiating rifampin. Further research is needed to clarify the optimal sequence of interventions that would lead to the highest rate of successful treatment.

\section{REFERENCES}

${ }^{1}$ Osmon DR, Berbari EF, Berendt AR, et al. Diagnosis and management of prosthetic joint infection: Clinical practice guidelines by the Infectious Diseases Society of America. Clin Infect Dis 2013; 56(1):el-e25. PMID: 23223583.

2 Tafer N, Belaieff W, Cuérel C, Zingg M, Hoffmeyer P. Uçkay I. Optimal diagnosis, prevention, and management of periprosthetic joint infection. Orthop Res Rev 2015; 7:11-19.

${ }^{3}$ Coiffier G, Albert JD, Arvieux C, Guggenbuhl P. Optimizing combination rifampin therapy for staphylococcal osteoarticular infections. Joint Bone Spine 2013; 80(1):11-17. PMID: 23332140.

${ }^{4}$ Liu C, Bayer A, Cosgrove SE, et al. Clinical practice guidelines by the Infectious Diseases Society of America for the treatment of methicillin-resistant Staphylococcus aureus infections in adults and children. Clin Infect Dis 2011; 52(3):el8-55. PMID: 21208910.

${ }^{5}$ Niska JA, Shahbazian JH, Ramos RI, Francis KP, Bernthal NM, Miller LS. Vancomycin-rifampin combination therapy has enhanced efficacy against an experimental Staphylococcus aureus prosthetic joint infection. Antimicrob Agents Chemother 2013; 57(10):5080-5086. PMID: 23917317.

${ }^{6}$ Achermann Y, Eigenmann K, Ledergerber B, et al. Factors associated with rifampin resistance in staphylococcal periprosthetic joint infections (PJI): A matched case-control study. Infection 2013; 41(2):431-437. PMID: 22987291

7 Peel TN, Buising KL, Dowsey MM, et al. Outcome of debridement and retention in prosthetic joint infections by methicillin-resistant staphylococci, with special reference to rifampin and fusidic acid combination therapy. Antimicrob Agents Chemother 2013; 57(1):350-355. PMID: 23114758.

${ }^{8}$ Chang YJ, Lee MS, Lee CH, Lin PC, Kuo FC. Daptomycin treatment in patients with resistant staphylococcal periprosthetic joint infection. BMC Infect Dis 2017; 17(1):736. PMID: 29187163.

9 Parvizi J, Azzam K, Ghanem E, Austin MS, Rothman RH. Periprosthetic infection due to resistant staphylococci: Serious problems on the horizon. Clin Orthop Relat Res 2009; 467(7):1732-1739. PMID: 19408061.
KANSAS JOURNAL of MEDICINE

RIFAMPIN RESISTANCE

continued.

${ }^{10}$ Lora-Tamayo J, Murillo O, Iribarren JA, et al. A large multicenter study of methicillin-susceptible and methicillin-resistant Staphylococcus aureus prosthetic joint infections managed with implant retention. Clin Infect Dis 2013; 56(2):182-194. PMID: 22942204.

Keywords: prosthesis-related infection, methicillin-resistant staphylococcus aureus, rifampin resistance protein, bacteria 